\title{
Exploring the relationship between the big five personality characteristics and dietary habits among students in a Ghanaian University
}

\author{
Freda Dzifa Intiful ${ }^{1 *}$ (D), Emefa Gifty Oddam ${ }^{1}$, Irene Kretchy ${ }^{2}$ and Joana Quampah ${ }^{1}$
}

\begin{abstract}
Background: Adherence to good dietary practices has been linked to disease prevention and better quality of life yet, University students are known to have poor dietary intake and diet quality. For an effective behaviour modification in dietary habits, an understanding of the association between an individual's personality traits and dietary habits are of much significance.

The aim of this study was to determine the relationship between personality traits and dietary habits among University students in Ghana.

Methods: A cross-sectional design involving 400 students was employed. Information on socio-demographic characteristics and Body Mass Index were obtained. The big 5 Personality traits (extraversion, conscientiousness, agreeableness, openness and neuroticism) was assessed using a 50-item International Personality Item Pool (IPIP) by Goldberg. The three factor eating questionnaire (TFEQ) was used to obtain further information on dietary habits.

Results: The majority of the students had high scores for conscientiousness. Except for neuroticism, all the personality traits had a significant association with at least one of the dietary habits explored. Extraversion was positively associated with neophagia $(p=0.028)$ and food interest $(p=0.008)$, conscientiousness was associated with variety $(p=0.045)$ and sugar moderation $(p=0.006)$, agreeableness was associated with neophagia $(p=0$. $005)$, skipping of meals $(p=0.007)$ and variety $(p=0.005)$ and openness associated with food interest $(p=0.009)$.

Conclusion: Personality traits showed associations with certain dietary habits but further studies are required to identify persons who are at risk of diet related diseases to inform the development of appropriate interventions.
\end{abstract}

Keywords: Dietary, Extraversion, Conscientiousness, Neuroticism, Agreeableness and openness, Students, Ghana

\section{Background}

Dietary habits refer to the set of choices or decisions one makes with regards to foods eaten. They involve what to eat, when to eat, how much to eat and where to eat [1]. These are affected by the taste preferences, variety in foods selected, frequency of meal consumption, portion sizes, snacking behaviour and skipping of meals. College students are known to have poor dietary intakes and diet quality.

\footnotetext{
*Correspondence: fdintiful@chs.edu.gh; fdintiful@ug.edu.gh

${ }^{1}$ School of Biomedical and Allied Health Sciences, Department of Dietetics,

University of Ghana, P.O. Box KB 143, Korle- Bu, Accra, Ghana

Full list of author information is available at the end of the article
}

Several factors such as changes in residence, time management or convenience, eating out, financial constraints, family influence, obsession to control weight and nutrition misconception have been associated with this trend of poor dietary habits [2,3].

There still remains a gap between dietary knowledge and actual dietary intake within the population such that people still find it difficult to change from negative dietary patterns to healthy options [4]. It has been postulated that the complex interaction between psychological, cultural, environmental and behavioural factors exerts an influence on an individual's ability to alter dietary habits [5].

(C) The Author(s). 2019 Open Access This article is distributed under the terms of the Creative Commons Attribution 4.0 International License (http://creativecommons.org/licenses/by/4.0/), which permits unrestricted use, distribution, and 
Understanding of the association between an individual's personality traits and food habits have been posited to be relevant for an effective behaviour modification in eating habits [6]. Some studies have shown an association between eating disorders, body weight and personality. One of such studies found dietary disinhibition to be strongly associated with adult weight gain. Furthermore, dietary restraints were reported to reduce this effect when dietary disinhibition was high [7]. Provencher and colleagues also observed various psychological factors to be associated with personality traits and some eating behaviours [8].

Personality traits are behavioral characteristics that are consistently expressed by a person or the distinct patterns exhibited in behaviour [9]. Contemporary personality psychologists widely agree that there are five core domains or dimensions of traits that interact to form personality and shape social landscape [10]. These personality traits are also known as the 'Big 5' or the 'Five Factor Model' personality traits [11]. The 'Five Factor Model' has been shown to account for different traits in personality without overlapping with other traits and has demonstrated consistency in interviews, self-descriptions and physical observations [12]. These traits are broadly categorized as extraversion, agreeableness, conscientiousness, neuroticism and openness to experience [12, 13]. Certain personality traits have been linked to weight among children. For instance, low conscientiousness and high impulsivity have been associated with high body mass index and unhealthy food choices among children $[1,14]$. Furthermore, a positive correlation was observed between psychoticism and unhealthy eating and neuroticism with pickiness and neophobia whereas neuroticism was negatively correlated with healthy eating and health habits [1].

The relationship between psychological factors such as one's emotions or personality trait and how they determine eating habits may still be relevant. In a review, Macht could argue the relationship between emotions and eating habits, positing that emotions could regulate eating, likewise eating may regulate one's emotions [15]. Also in a more recent article, authors were able to establish that the big five personality traits could be a useful tool in identifying people that may have challenges living with food allergies [16].

Knowledge of the influence of personality trait on dietary habit is of relevance in health promotion and individualizing dietary health care plan and not a one size fits all approach [8, 17]. Individualized interventions take into account demographic characteristics such as sex and age, culture and beliefs, eating practices and in rare circumstances, personality attributes [18]. Some studies have examined the links between personality attributes and eating practices among adolescents and college students $[1,18$, 19]. Also, the interaction between gender, personality traits and dietary habits is under explored particular in Africa.
Elsewhere, some studies have shown males exhibiting poor dietary habits compared to females [20, 21]. There is also evidence establishing the interaction between personality traits, gender and eating habits. Among Norwergian children, girls with lower conscientiousness and higher neurotism were more likely to consume sweet drinks [12]. Therefore researching into personality traits of college students may provide more insight into the determinants of diet quality [19]. However research in the area of personality traits and diet is highly under explored in Ghana. This study therefore aimed at determining the relationship between personality traits and dietary habits among college students in a Ghanaian tertiary academic institution.

\section{Methods}

\section{Study design and area}

The study was a descriptive school-based cross-sectional study conducted among college students at the University of Ghana.

\section{Study participants and sample size}

The study included undergraduate students of the University of Ghana who consented to be part of the study. Respondents included both resident and non-residential students. Pregnant women and students whose anthropometric measurements could not be taken easily were excluded from the study. Based on a 95\% Confidence interval, precision of $5,50 \%$ probability and an attrition rate of $4 \%$, a sample size of 403 was obtained but eventually 400 participants consented to be part of the study.

\section{Sampling}

The sampling followed a multistage approach. Students were recruited from the four main colleges in the University i.e. the Colleges of Health, Education, Humanities, and Basic and Applied Sciences. Balloting was used to select two colleges out the total of four. Eight departments were further selected through the same process of balloting from the two schools earlier selected. Students in the departments were randomly selected and then approached to be part of the study.

\section{Ethical consideration}

The study was approved by the School of Biomedical and Allied Health Sciences Ethics and Protocol Review Committee with the code number SBAHS-ET./10,443,580/AA/ 6A/2012-2013. A written informed consent was obtained from each participant before data collection.

\section{Measurements}

Data were obtained using a structured, self-administered questionnaire. Data gathered included socio-demography, body weight, height and Body Mass Index (BMI), following standard procedures. Personality traits were assessed 
using a 50- item International Personality Item Pool -(IPIP) tool [22]. The 50-item IPIP tool measures the markers of the big five factor structure reported by Goldberg [23]. A “Three-Factor Eating Questionnaire” (TFEQ) was used to assess three aspects of the dietary habits which are Cognitive restraint of food intake involving monitoring and control of food intake and body weight; Disinhibition of control of eating which involves tendency to continue eating even when satiated, hunger or emotional eating i.e. finding solace in food when stressed or reaction to external cues $[24,25]$. The TFEQ comprised of 18 items. Nine (9) of the items focused the control of food intake and body weight, six (6) concerned with disinhibition of control of eating and the remaining three (3) on emotional eating. Participants had to choose among four responses which appropriately relates to them most. Each of the four (4) responses followed a scoring system. The total score ranged between 18 and 72 . The higher the score the more depended upon a particular dietary behaviour.

Additional dietary information was taken using a structured questionnaire which assessed pickiness (being fussy, choosy or selective with regards to food), 'neophagia' (acceptance of new and unusual foods such as foods from other cultures), food interest (having strong liking for food as compared to one who finds having to eat as a bother and would only eat because he/she has to eat), variety seeking, skipping of meals, consumption of fiber, consumption of fruits and vegetables as well as intake of fats, sugar and salt. Students were asked to indicate the strength of their agreement with specific statements pertaining to the dietary habits on a true or false scale which was expanded to definitely true, mostly true, mostly false and definitely false. False for a reversed question was taken as true (compare 'I like to stick to the foods that I know' to 'I enjoy trying new foods'). A number of statements were analyzed (based on this true or false scale) to determine whether or not the respondent was prone to the dietary habit in question.

The International Personality Item Pool (IPIP) is a validated tool for assessing the personality domains of conscientiousness, openness, neuroticism, extraversion and agreeableness [17]. This comprehensive 50-item self-assessment personality test instrument measures the strength of these five fundamental dimensions of personality. Respondents were given a list of statements concerning their perception of themselves in a variety of situations and were to choose from a scale answers that most closely reflected their attitude by indicating the strength of their agreement with each statement. Although not indicated on the actual survey questionnaire, there were numbers in parentheses after each IPIP scale item indicating the type of personality factor being measured, i.e. (1) Extraversion, (2) Agreeableness, (3) Conscientiousness, (4) Emotional Stability, and (5) Openness, as well as the direction of scoring the scale of $1-5$ (i.e. positive or negative). The negatively keyed items were reverse scored. A sum of all the values of the scale items was obtained to give the total scale score once the numbers were assigned for all of the items. The individual's personality traits of extraversion, openness, neuroticism and conscientiousness were then calculated based on their responses. An individual at or above the fiftieth percentile in a particular trait was considered to be high in that trait. Scores below the fiftieth percentile were considered low. This categorization was done to clearly depict where personality strengths and weaknesses of the participants fall.

\section{Data analysis}

Data collected were analyzed using SPSS version 20.0. Data were summarized using percentages, means and standard deviations. Chi-square analysis was used to test for association between measured dietary habits and Body Mass Index (BMI) and dietary habits and personality traits. Independent T-test was performed for differences between the scores obtained female and the male students. The level of significance was set at $p \leq 0.05$. Cronbach's alpha was used to test for the reliability of the questionnaires that were used.

\section{Results \\ Demographic and BMI characteristics of students}

Table 1 describes the demographic characteristics of the students. A total of 400 students participated comprising of $230(57.5 \%)$ males and $170(42.5 \%)$ females. The mean age of the students was $21.19 \pm 1.96$ years. The majority of the students were in the 2 nd and 3rd years. The majority of the respondents were within the normal range of BMI (72.8\%). About $15 \%$ of them were overweight or obese. However more females were overweight (17.8\%) or obese $(4.1 \%)$ than their male counterparts (8.3 and $2.6 \%$ respectively). An independent $t$-test performed showed no significant differences in the BMI's between the males and females.

Assessment of their personality traits showed that more than half of the respondents had high scores for conscientiousness $(73.2 \%)$ and agreeableness $(51.5 \%)$ and low scores for extraversion (63\%), neuroticism (70\%) and openness $(79.3 \%)$. Both males and females showed similar trends with their personality traits. (Fig. 1).

\section{Reliability statistics of tests}

The subscales for measuring personality traits, each consisting of 10 items were found to be reliable (extraversion $\alpha=0.712$, agreeableness $\alpha=0.698$, openness $\alpha=$ 0.738, neuroticism $\alpha=0.740$, conscientiousness $\alpha$ $=0.724)$. Cronbach's alpha for three (3) items under each of the subscales for dietary habits were 0.754 (cognitive restraint), 0.740 (disinhibition) and 0.780 (emotional eating). The rest ranged between 0.668 and 0.782 . 
Table 1 Demographic characteristics of respondents $(N=400)$

\begin{tabular}{|c|c|c|c|c|}
\hline Variables & $\begin{array}{l}\text { Male (230) } \\
n(\%)\end{array}$ & $\begin{array}{l}\text { Female (170) } \\
n(\%)\end{array}$ & $\begin{array}{l}\text { Total } \\
\text { N (\%) }\end{array}$ & $P$-value \\
\hline $\begin{array}{l}\text { Age }(\text { Mean } \pm S D) \\
\text { years }\end{array}$ & $\begin{array}{l}21.50 \pm \\
2.04\end{array}$ & $20.76 \pm 1.77$ & $\begin{array}{l}21.19 \pm \\
1.96\end{array}$ & $0.008^{*}$ \\
\hline \multicolumn{5}{|l|}{ Year of study } \\
\hline 1 & $50(21.7)$ & 38 (22.4) & $88(22.0)$ & \\
\hline 2 & $63(27.0)$ & 47 (27.6) & 110 (27.5) & \\
\hline 3 & 55 (23.9) & $54(31.2)$ & 109 (27.3) & \\
\hline 4 & $62(26.9)$ & 31 (18.2) & $93(23.3)$ & \\
\hline $\begin{array}{l}\text { BMI }\left(\mathrm{kg} / \mathrm{m}^{2}\right) \\
\text { Mean } \pm \mathrm{SD}\end{array}$ & $\begin{array}{l}21.80 \pm \\
3.28\end{array}$ & $22.41 \pm 3.63$ & $\begin{array}{l}22.06 \pm \\
3.44\end{array}$ & 0.05 \\
\hline Underweight & 29 (12.7) & $17(10.1)$ & 46 (11.5) & \\
\hline Normal & $175(76.1)$ & $115(68.0)$ & 291 (72.8) & \\
\hline Overweight & $19(8.3)$ & 30 (17.8) & 49 (12.3) & \\
\hline Obese & $6(2.6)$ & $7(4.1)$ & $13(3.3)$ & \\
\hline
\end{tabular}

\section{Dietary habit traits of students}

Table 2 shows the percentage of students exhibiting the various dietary traits and the $P$-values obtained after conducting an independent $\mathrm{T}$-test for differences between the scores obtained by the females and the males. Significant differences were observed between the males and females in the areas of emotional eating, pickiness, neophagia, fiber consumption, sugar and salt moderation. A significant proportion of females than males reported being emotional eaters, picky eaters, practicing neophagia and consuming fiber-rich foods. The proportion of male respondents reporting moderate sugar intake was significantly higher than the proportion observed in females. No differences were observed with salt intake between males and females.
Relationship between personality traits, dietary habits and BMI

The association between the personality traits, dietary habits and BMI of the students are shown on Tables 3 and 4. No significant association was observed between BMI and dietary habits, however pickiness was positively associated with waist-to-hip ratio in both males and females. In determining the association between personality trait and dietary habits, extraversion $(p=0.028)$, agreeableness $(p=$ $0.045)$ and openness $(p=0.009)$ were all significantly linked to neophagia. Extraversion was significantly associated with food interest $(p=0.008)$, conscientiousness significantly associated with variety $(\mathrm{p}=0.045)$, agreeableness was also associated with skipping meals $(p=0.007)$ and conscientiousness associated with moderate sugar intake $(p=0.006)$.

\section{Discussion}

This study reports on an understudied area of nutrition research in Ghana where the relationship between an individual's personality trait and dietary habits were investigated. The majority of the students had high scores on conscientiousness describing themselves in ways that portray self-discipline, dutifulness and planned behaviour as compared to disorderliness. Some trait characteristics for individuals having high scores for conscientiousness include being practical, thorough, neat, efficient, systematic and careful [26]. It is therefore commendable to have a high number of students exhibiting this trait. A slightly higher number of females were found to have high levels of conscientiousness in this study. This agrees with findings that this trait is exhibited more in females than males [27]. About 51.5\% scored high on agreeableness in this study. This finding contradicts the findings of Cho et al. [28] where more of the respondents scored higher on agreeableness compared to the other traits. Individuals

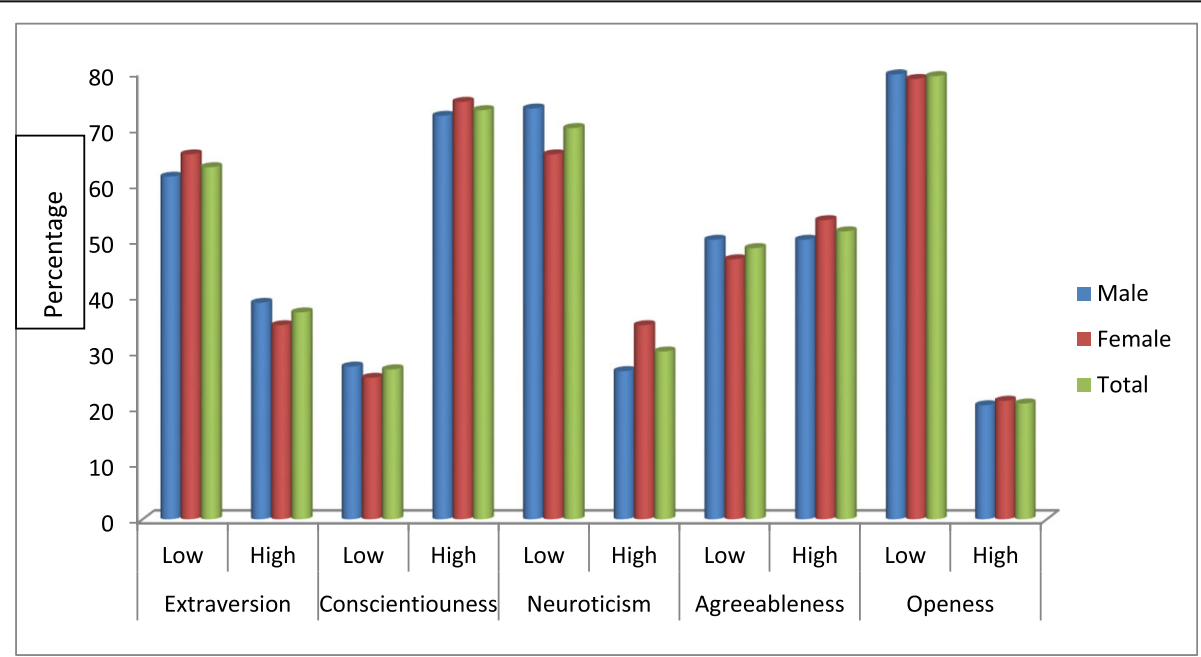

Fig. 1 Personality trait of students 
Table 2 Table showing the dietary habits of students according to gender

\begin{tabular}{lllll}
\hline & Total & Males & Females & $P$ - value \\
\hline Disinhibition & 19.0 & 20.9 & 16.5 & 0.635 \\
Cognitive Restraint & 25.2 & 21.7 & 30 & 0.060 \\
Emotional eating & 19.5 & 15.7 & 24.7 & $0.024^{*}$ \\
Pickiness & 29.0 & 24.8 & 34.7 & $0.031^{*}$ \\
Neophagia & 33.8 & 28.7 & 40.6 & $0.013^{*}$ \\
Food interest & 47.5 & 47.4 & 47.6 & 0.960 \\
Variety & 43.8 & 42.6 & 45.3 & 0.593 \\
Skipped meals & 44.0 & 43 & 45.3 & 0.654 \\
Fiber consumption & 23.2 & 19.1 & 28.8 & $0.023^{*}$ \\
Fruits and vegetables & 58.2 & 56.5 & 60.5 & 0.415 \\
Fats moderation & 45.8 & 44.3 & 47.6 & 0.513 \\
Sugar moderation & 36.2 & 42.4 & 31.7 & $0.029^{*}$ \\
Salt moderation & 45.5 & 49.6 & 40 & 0.058 \\
\hline
\end{tabular}

with high scores for agreeableness can be described as kind, sympathetic, trustful, cooperative and considerate [27]. The differences in scores could be attributed to the category of people in the different studies. This study was mainly among young adults whiles Cho et al. [28] focused on adolescents. Because adolescents are still mainly under parental control, it is likely that they may tend to exhibit the characteristics of agreeableness as explained earlier.

More males than females scored high on neuroticism. This also contradicts some studies $[29,30]$ in which female participants had high scores for neuroticism and agreeableness suggesting a more emotional and sociable personality compared to the male participants who had high scores in extraversion, conscientiousness and openness. Fischer et al. [30] also revealed that women tend to
Table 4 Association between dietary habits and BMl

\begin{tabular}{ll}
\hline Dietary Habits & BMI P-values \\
\hline Disinhibition & 0.918 \\
Conscientiousness & 0.382 \\
Emotional eating & 0.686 \\
Pickiness & 0.941 \\
Neophagia & 0.676 \\
Food Interest & 0.200 \\
Variety & 0.847 \\
Skipping Meals & 0.825 \\
Fibre Intake & 0.501 \\
Fruits and Vegetables & 0.703 \\
Fat moderation & 0.255 \\
Sugar moderation & 0.050 \\
Salt moderation & 0.120
\end{tabular}

report more negative emotionality than men which in turn affect their dietary habits.

In this study, neuroticism was not significantly associated with any of the dietary habits. This is in contrast with other similar studies in which neuroticism was associated with dietary habits such as pickiness, neophobia, breakfast skipping and promotion of other unhealthy food choices $[1,29,31]$. In yet another study, it was reported that lower scores of neuroticism were associated with making healthy dietary choices [32]. It is difficult to explain in the present study why neuroticism was not significantly associated with any of the dietary habits. Conscientiousness was associated with variety and sugar moderation. In other similar studies, conscientiousness was linked to healthy eating behaviors such as avoidance of sweets, confectionaries and consumption of fruits [29. 31], regular eating time and

Table 3 Association between personality traits and dietary habits of students

\begin{tabular}{|c|c|c|c|c|c|}
\hline & Extraversion & Conscientiousness & Neuroticism & Agreeableness & Openness \\
\hline Disinhibition & 0.198 & 0.702 & 0.541 & 0.135 & 0.699 \\
\hline Cognitive Restraint & 0.531 & 0.996 & 0.353 & 0.359 & 0.579 \\
\hline Emotional eating & 0.455 & 0.805 & 0.205 & 0.768 & 0.800 \\
\hline Pickiness & 0.177 & 0.323 & 0.665 & 0.870 & 0.985 \\
\hline Neophagia & $0.028^{*}$ & 0.243 & 0.908 & $0.045^{*}$ & $0.009^{*}$ \\
\hline Food Interest & $0.008^{*}$ & 0.968 & 0.827 & 0.528 & 0.887 \\
\hline Variety & 0.273 & $0.045^{*}$ & 0.582 & $0.005^{*}$ & 0.675 \\
\hline Skipping meals & 0.220 & 0.066 & 0.629 & $0.007^{*}$ & 0.713 \\
\hline Fiber & 0.171 & 0.764 & 0.588 & 0.092 & 0.780 \\
\hline Fruits & 0.965 & 0.126 & 0.177 & 0.310 & 0.679 \\
\hline Fat moderation & 0.634 & 0.642 & 0.196 & 0.310 & 0.213 \\
\hline Sugar moderation & 0.249 & $0.006^{*}$ & 0.734 & 0.248 & 0.780 \\
\hline Salt moderation & 0.367 & 0.197 & 0.148 & 0.957 & 0.850 \\
\hline
\end{tabular}


avoidance of salty foods [33] with individuals having high scores for conscientiousness being more receptive to dietary advice and adoption of healthful practices [27, 30]. Personality traits have also been reported to correlate with dietary habits in the following descending order; conscientiousness, extraversion, agreeableness, emotional stability and openness [33]. In this study, extraversion was positively associated with food interest and neophagia. MacNicol et al., associated food interest with unhealthy eating and extraversion [1]. Extraverts are individuals who are warm and sociable and would not only stick to the foods they know but would like to try new foods from other cultures. Extraverts also have high food interest. They do not find having to eat to be a bother or only eat because they have to eat but they express much liking for food. Conscientiousness was associated with variety seeking and moderation in salt intake. This shows the students who were disciplined, industrious and dutiful also sought variety in their diet and moderated salt intake. Other reports suggest that conscientiousness was associated with fat moderation as opposed to extraversion [17]. Agreeableness was associated with neophagia, variety seeking and skipping of meals. This suggests that those who like to modify their character to suit others also like to try new foods but tend to skip meals which are not encouraged. In a contradictory finding, Kikuchi \& Watanabe [29] observed desired traits such as avoidance and dislike of salty foods, desire to be healthy and avoidance of animal fat and burnt food in individuals with high scores for agreeableness [30].

This study could have been influenced by some limitations. The assessment of personality traits and dietary habits among the students were based on the individual's own assessment of his/her self and therefore responses could be biased. Additionally reported eating habits of students could also be influenced by their purchasing power as well as other challenges such as time, availability of food on campus and the demands of their academic work.

To the best of our knowledge, this study is a new ground in Ghana. With the increase in the prevalence of chronic diseases as a result of changing dietary habits, the need to explore relevant ways to improve dietary habits is important. This calls for interventions tailored to the individual. Therefore the need to explore personality traits of individuals that affect dietary habits cannot be overemphasized. This study is therefore relevant in spite of the total dependence on respondents to be objective in answering questions about their personality traits.

\section{Conclusion}

Personality traits have been shown to be associated with dietary habits but further studies are required to identify persons who are at risk of diet related diseases to inform the development of appropriate dietary interventions bearing in mind the personality traits they exhibit.

\section{Acknowledgements}

Authors wish to thank all University of Ghana students who participated in this study.

\section{Funding}

Not applicable.

\section{Availability of data and materials}

All non-identifying data are available upon request to the corresponding author.

\section{Authors' contributions}

$\mathrm{Fl}$ and IK conceptualized the study design. EO, FI, IK and JQ participated in data collection and analysis. FI, IK, JQ and EO wrote the manuscript. All authors read and approved the final manuscript.

\section{Ethics approval and consent to participate}

Ethical approval was obtained from the Protocol and Ethics Review committee of the School of Biomedical and Allied Health Sciences, University of Ghana. The reference number is SBAHS-ET./10,443,580/AA/6A/2012-2013. Written informed consent was sought from all participating students.

\section{Consent for publication}

Not applicable.

\section{Competing interests}

Authors declare no competing interests.

\section{Publisher's Note}

Springer Nature remains neutral with regard to jurisdictional claims in published maps and institutional affiliations.

\section{Author details}

${ }^{1}$ School of Biomedical and Allied Health Sciences, Department of Dietetics, University of Ghana, P.O. Box KB 143, Korle- Bu, Accra, Ghana. ${ }^{2}$ School of Pharmacy, Department of Pharmacy Practice and Clinical Pharmacy, University of Ghana, Accra, Ghana.

Received: 1 June 2017 Accepted: 18 February 2019

Published online: 22 February 2019

\section{References}

1. MacNicol SA, Murray SM, Austin EJ. Relationships between personality, attitudes and dietary behaviour in a group of Scottish adolescents. Personal Individ Differ. 2003;35(8):1753-64.

2. Ganasegeran K, Al-Dubai SAR, Qureshi AM, Al-abed AA, Rizal AM, Aljunid SM. Social and psychological factors affecting eating habits among university students in a Malaysian medical school: a cross-sectional study. BMC Nutr Journal. 2012;11(1):48.

3. Deliens T, Clarys P, De Bourdeaudhuij I, Deforche B. Determinants of eating behaviour in university students: a qualitative study using focus group discussions. BMC Public Health. 2014;14:53.

4. van den Bree MB, Przybeck M, Robert Cloninger TR. Diet and personality: associations in a population-based sample. Appetite. 2006;46(2):177-88.

5. Brownell KD, Cohen LR. Adherence to dietary regimens 1: an overview of research. Behav Med. 1995;20(4):149-54.

6. Fassino S, Leombruni P, Pierò A, Daga GA, Amianto F, Rovera G, Rovera GG. Temperament and character in obese women with and without binge eating disorder. Compr Psychiatry. 2002:43(6):431-7.

7. Hays NP, Bathalon GP, McCrory MA, Roubenoff R, Lipman R, Roberts SB. Eating behavior correlates of adult weight gain and obesity in healthy women aged 55-65 y. Am J Clin Nutr. 2002;75(3):476-83.

8. Provencher V, Bégin C, Gagnon-Girouard M-P, Tremblay A, Boivin S, Lemieux S. Personality traits in overweight and obese women: associations with BMI and eating behaviors. Eat Behav. 2008;9(3):294-302.

9. Schacter DL, Gilbert DT. Wegner DM Psychology. 2nd ed. New York: Worth; 2011

10. Matthews G, Deary IJ, Whiteman MC. Personality traits. 2009. Cambridge University Press.

11. Digman JM. Personality structure: emergence of the five-factor model. Annu Rev Psychol. 1990;41(1):417-40. 
12. McCrae RR, Terracciano A. Personality profiles of cultures: aggregate personality traits. J Pers Soc Psychol. 2005;89(3):407-25.

13. Vollrath ME, Hampson SE, Júlíusson PB. Children and eating. Personality and gender are associated with obesogenic food consumption and overweight in 6- to 12-year-olds. Appetite. 2012;58(3):1113-7.

14. Braet $C$, Claus L, Verbeken S, Van Vlierberghe L. Impulsivity in overweight children. European Child \& Adolescent Psychiatry. 2007;16(8):473-83.

15. Macht M. How emotions affect eating: a five-way model. Appetite. 2008: 50(1):1-11. https://doi.org/10.1016/j.appet.2007.07.002.

16. Conner TS, Mirosa M, Bremer P, Peniamina R. The role of Personality in daily food allergy experiences. Front Psychol. 2018;9(29). https://doi.org/10.3389/ fpsyg.2018.00029.

17. Goldberg LR, Strycker LA. Personality traits and eating habits: the assessment of food preferences in a large community sample. Personal Individ Differ. 2002:32(1):49-65.

18. Raynor DA, Levine H. Associations between the five-factor model of Personality and health behaviors among college students. J Am Coll Heal. 2009;58(1):73-82.

19. Horacek TM, Betts NM. College students' dietary intake and quality according to their Myers Briggs type Indicator Personality preferences. J Nutr Educ. 1998;30(6):387-95.

20. Caine-Bish NL, Scheule B. Gender differences in food preferences of schoolaged children and adolescents. J Sch Health. 2009;79(11):532-40.

21. Sweeting HN. Gendered dimensions of obesity in childhood and adolescence. Nutr J. 2008;7(1):1. https://doi.org/10.1186/1475-2891-7-1.

22. Goldberg LR. International Personality item Pool: a scientific collaboratory for the development of advanced measures of personality and other individual differences. 1999. Available at ipip. ori. org/ipip/.

23. Administering IPIP measures, with a 50-item sample questionnaire. Retrieved from https://ipip.ori.org/New_IPIP-50-item-scale. htm\#SampleQuestionnaire

24. Kavazidou E, Proios M, Liolios I, Doganis G, Petrou K, Tsatsoulis A, Tsiligiroglou-Fachantidou A. Structure validity of the three-factor eating questionnaire-R18 in Greek population. Journal of human sports and exercise. 2012;7(1).

25. The three factor eating questionnaire. Retrieved from http://www.med. umich.edu/pdf/weight-management/TFEQ-r18.pdf

26. Lunn TE, Nowson CA, Worsley A, Torres SJ. Does personality affect dietary intake? Nutrition. 2014;30(4):403-9.

27. Cho MS, Kim M, Cho W. Relationships of adolescent's dietary habits with personality traits and food neophobia according to family meal frequency. Nutr Res Pract. 2014;8(4):476-81. https://doi.org/10.4162/nrp.2014.8.4.476.

28. Kikuchi Y, Personality WS, Habits D. Journal of Epidemiology. 2000;10(10):191-8.

29. Fischer AH, Rodriguez Mosquera PM, van Vianen AEM, Manstead ASR (2004). Gender and culture differences in emotion. Emotion. 2004. 4(1): 87-94.

30. Keller C, Siegrist M. Does personality influence eating styles and food choices? Direct and indirect effects. Appetite. 2015;84:128-38. https://doi. org/10.1016/j.appet.2014.10.003

31. Mottus R, Johnson W, Starr JM. Dearya IJ. Correlates of personality trait levels and their changes in very old age: the Lothian birth cohort 1921. J Res Pers. 2012;46(3):271-8.

32. Kikuchi Y, Inoue T, Ito M, Masuda M, Yoshimura K, Watanabe S. Health consciousness of young people in relation to their Personality. Journal of Epidemiology. 1999;9:121-31.

33. Bae SJ. In: a study on relationship between eating habit and personality or scholastic adjustment of middle school student [master's thesis]. Seoul: Korea University; 2004

Ready to submit your research? Choose BMC and benefit from:

- fast, convenient online submission

- thorough peer review by experienced researchers in your field

- rapid publication on acceptance

- support for research data, including large and complex data types

- gold Open Access which fosters wider collaboration and increased citations

- maximum visibility for your research: over $100 \mathrm{M}$ website views per year

At BMC, research is always in progress.

Learn more biomedcentral.com/submissions 